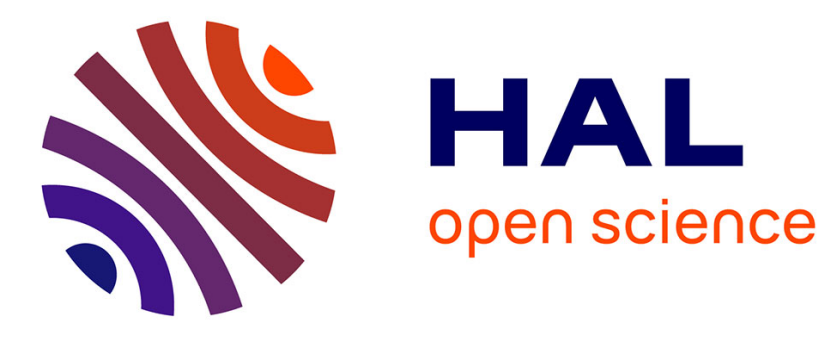

\title{
Electrical characterization of all-optical helicity-dependent switching in ferromagnetic Hall crosses
}

M S El Hadri, P. Pirro, C.-H Lambert, N. Bergeard, S. Petit-Watelot, Michel Hehn, G. Malinowski, F. Montaigne, Y. Quessab, R Medapalli, et al.

\section{To cite this version:}

M S El Hadri, P. Pirro, C.-H Lambert, N. Bergeard, S. Petit-Watelot, et al.. Electrical characterization of all-optical helicity-dependent switching in ferromagnetic Hall crosses. Applied Physics Letters, 2016, 108, pp.92405 - 153905. 10.1063/1.4943107. hal-01686954

\section{HAL Id: hal-01686954 \\ https://hal.univ-lorraine.fr/hal-01686954}

Submitted on 17 Jan 2018

HAL is a multi-disciplinary open access archive for the deposit and dissemination of scientific research documents, whether they are published or not. The documents may come from teaching and research institutions in France or abroad, or from public or private research centers.
L'archive ouverte pluridisciplinaire HAL, est destinée au dépôt et à la diffusion de documents scientifiques de niveau recherche, publiés ou non, émanant des établissements d'enseignement et de recherche français ou étrangers, des laboratoires publics ou privés. 


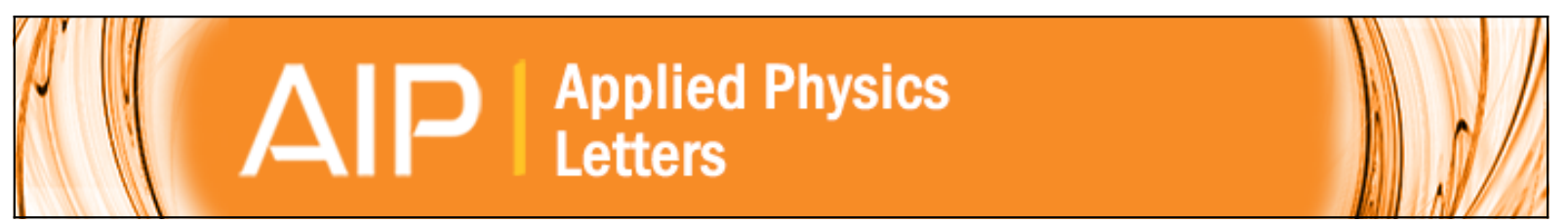

Electrical characterization of all-optical helicity-dependent switching in ferromagnetic Hall crosses

M. S. El Hadri, P. Pirro, C.-H. Lambert, N. Bergeard, S. Petit-Watelot, M. Hehn, G. Malinowski, F. Montaigne, Y. Quessab, R. Medapalli, E. E. Fullerton, and S. Mangin

Citation: Applied Physics Letters 108, 092405 (2016); doi: 10.1063/1.4943107

View online: http://dx.doi.org/10.1063/1.4943107

View Table of Contents: http://scitation.aip.org/content/aip/journal/apl/108/9?ver=pdfcov

Published by the AIP Publishing

\section{Articles you may be interested in}

Ferromagnetic resonance of exchange-coupled perpendicularly magnetized bilayers

J. Appl. Phys. 119, 153905 (2016); 10.1063/1.4947227

Microscopic model for all optical switching in ferromagnets

Appl. Phys. Lett. 108, 142405 (2016); 10.1063/1.4945660

Magnetoresistance in Co/Pt based magnetic tunnel junctions with out-of-plane magnetization

J. Appl. Phys. 103, 07A918 (2008); 10.1063/1.2838282

Nucleation and propagation of domains walls in a $\mathrm{Co} / \mathrm{Pt}$ multilayer wire

J. Appl. Phys. 101, 09F508 (2007); 10.1063/1.2710224

Magnetization switching induced by in-plane current with low density in $\mathrm{Pt} / \mathrm{Co} / \mathrm{Pt}$ sandwich

J. Appl. Phys. 99, 08 G518 (2006); 10.1063/1.2175822

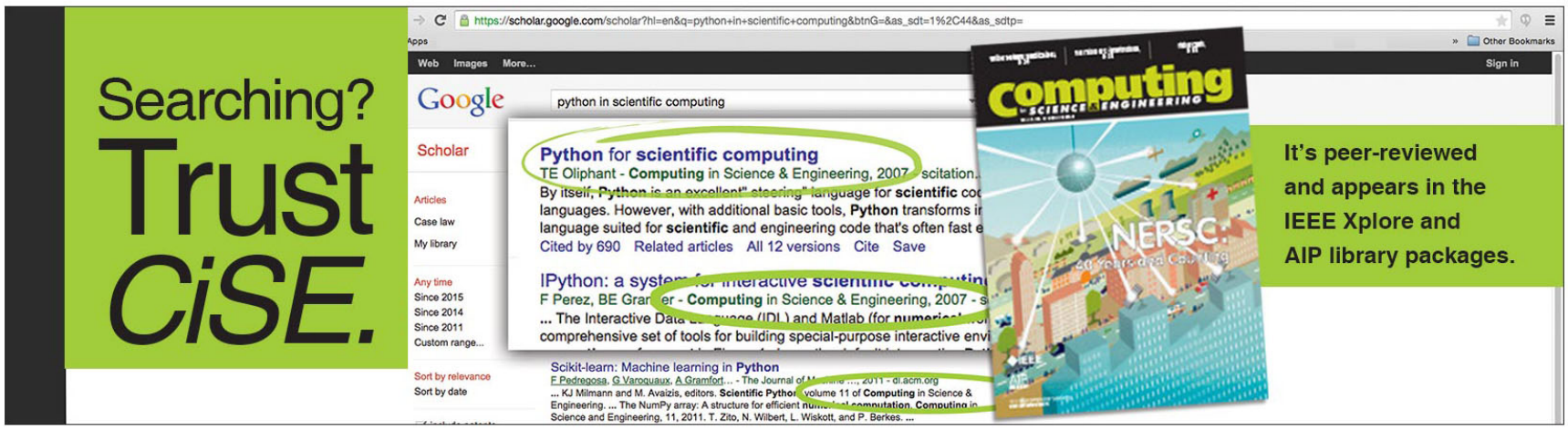




\title{
Electrical characterization of all-optical helicity-dependent switching in ferromagnetic Hall crosses
}

\author{
M. S. El Hadri, ${ }^{1}$ P. Pirro, ${ }^{1}$ C.-H. Lambert, ${ }^{1}$ N. Bergeard, ${ }^{1}$ S. Petit-Watelot, ${ }^{1}$ M. Hehn, ${ }^{1}$ \\ G. Malinowski, ${ }_{1}^{1}$ F. Montaigne, ${ }^{1}$ Y. Quessab, ${ }^{1,2}$ R. Medapalli, ${ }^{2}$ E. E. Fullerton, ${ }^{2}$ \\ and S. Mangin ${ }^{1}$ \\ ${ }^{1}$ Institut Jean Lamour, UMR CNRS 7198, Université de Lorraine, BP 70239, F-54506 Vandoeuvre-lès-Nancy, \\ France \\ ${ }^{2}$ Center for Magnetic Recording Research, University of California San Diego, La Jolla, \\ California 92093-0401, USA
}

(Received 12 November 2015; accepted 19 February 2016; published online 3 March 2016)

\begin{abstract}
We present an experimental study of all-optical helicity-dependent switching (AO-HDS) of ferromagnetic $\mathrm{Pt} / \mathrm{Co} / \mathrm{Pt}$ heterostructures with perpendicular magnetic anisotropy. The sample is patterned into a Hall cross and the AO-HDS is measured via the anomalous Hall effect. This all-electrical probing of the magnetization during AO-HDS enables a statistical quantification of the switching ratio for different laser parameters, such as the threshold power to achieve AO-HDS and the exposure time needed to reach complete switching at a given laser power. We find that the AO-HDS is a cumulative process, a certain number of optical pulses is needed to obtain a full and reproducible helicity-dependent switching. The deterministic switching of the ferromagnetic $\mathrm{Pt} / \mathrm{Co} / \mathrm{Pt}$ Hall cross provides a full "opto-spintronic device," where the remanent magnetization can be all-optically and reproducibly written and erased without the need of an external magnetic field. (C) 2016 AIP Publishing LLC. [http://dx.doi.org/10.1063/1.4943107]
\end{abstract}

Recent experiments have shown the capability to switch magnetization using ultrashort laser pulses without applying an external magnetic field. ${ }^{1,2}$ Initially shown for GdFeCo alloy films, all-optical switching (AOS) of this material was purely thermal and ultrafast, due to the different dynamics of $\mathrm{Gd}$ and FeCo sublattices. ${ }^{3,4}$ However, all-optical switching of GdFeCo alloy films is helicity dependent only for a narrow fluence range and the microscopic origin is explained by magnetic circular dichroism. ${ }^{5-7}$ Later, all-optical switching has also been discovered in other ferrimagnetic materials, ${ }^{8-10}$ and also in single ferromagnetic films. ${ }^{11}$ However, in these materials, all-optical switching turns out to be helicity dependent for a wide range of fluences, and its microscopic origin is still under debate.

Despite the fact that the microscopic mechanism for all-optical helicity-dependent switching (AO-HDS) is still under debate, these experiments helped pave the way for a new field combining optics and spintronics. ${ }^{12}$ This field called opto-spintronics makes use of the fact that both electrons and photons can carry a spin momentum. Since spins can be exchanged between the different carriers, this field is promising for fundamental research ${ }^{13-15}$ as well as for applications. ${ }^{16,17}$ For instance, all-optical switching could be implemented in spintronic devices like spin-transfer torque based memories ${ }^{18,19}$ or heat-assisted magnetic recording. ${ }^{20,21}$ It would combine speed and energy efficiency since ultrafast pulses are used instead of magnetic fields in today's technology. Towards this goal, we probe AO-HDS with pure magneto-transport read-out, thus combining optics and classical spintronics. The materials used are ferromagnetic multilayers showing strong perpendicular anisotropy, which are known to exhibit strong thermal stability. ${ }^{22}$

It was recently reported that $\mathrm{GdFeCo}$ based Hall crosses can be switched using ultrashort laser pulses. ${ }^{23}$ However, as the switching process in these devices was helicity independent and not optically reversible, the written data could not be changed or erased without using magnetic fields. This problem underlines the importance of helicity dependent switching because it enables the deterministic all-optical writing and erasing of data without applying any magnetic field.

In this study, we demonstrate the AO-HDS of ferromagnetic $\mathrm{Pt} / \mathrm{Co} / \mathrm{Pt}$ microstructures by an all-electrical measurement of the anomalous Hall effect. ${ }^{24}$ We show that using ultrafast femtosecond laser pulses, data in the form of the remanent magnetization can be reproducibly written and erased by changing the circular polarization of the pulses. This is demonstrated with both a static and a swept laser spot.

The investigated ferromagnetic sample is a multilayer thin film of Glass/Ta(3 nm)/Pt(3.7 nm)/Co( $(0.6 \mathrm{~nm}) / \operatorname{Pt}(3.7 \mathrm{~nm})$ grown by DC magnetron sputtering. The film shows a strong perpendicular magnetic anisotropy, with a first order anisotropy constant $K_{1}=2.65 \times 10^{6} \mathrm{~J} / \mathrm{m}^{3}$ and a saturation magnetization $M_{s}=1483 \mathrm{kA} / \mathrm{m}$. Thus, the effective perpendicular anisotropy constant is $K_{\text {eff }}=K_{1}-\frac{\mu_{0}}{2} M_{s}^{2}$ is positive, which leads to a perpendicular magnetization in remanence with a room-temperature coercive field of $\mu_{0} H=8 \mathrm{mT}$. The sample is then patterned using optical lithography to obtain a Hall cross with a 5- $\mu \mathrm{m}$ width (see Fig. 1). A DC current is injected along the $\mathrm{x}$ direction, and the Hall voltage is measured along the $y$ direction with the aim of quantifying the net z-component of magnetization within the Hall-cross area. For the experiments discussed here, no magnetic field is applied so the measured Hall voltage can be expressed using the Hall resistivity as follows:

$$
V_{\mathrm{AHE}}=\frac{\rho_{\mathrm{xy}} I}{t} \quad \text { with } \quad \rho_{\mathrm{xy}}=R_{\mathrm{AHE}} \vec{M} \hat{e}_{\mathrm{z}} .
$$




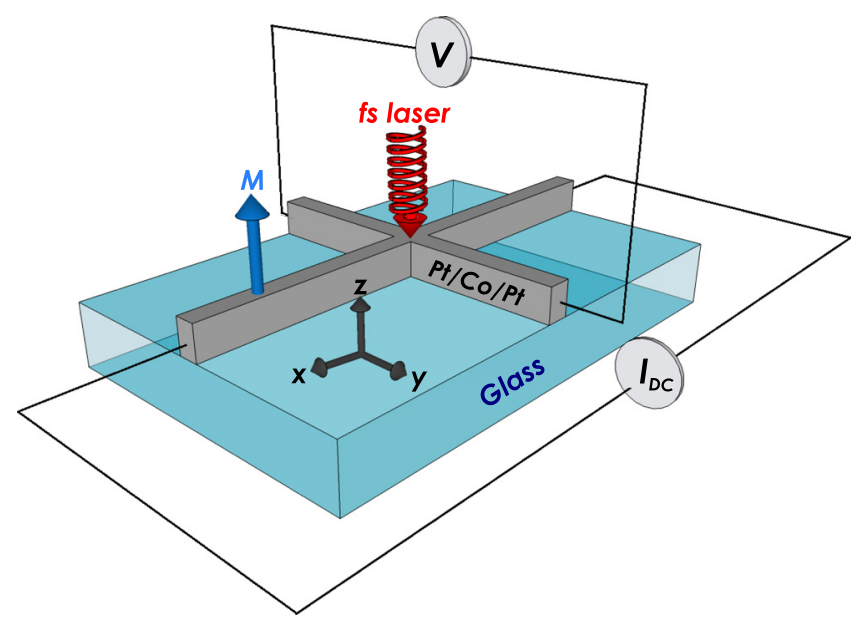

FIG. 1. Experimental set-up schematic: In a $5 \mu \mathrm{m}$ wide Hall cross of a ferromagnetic $\mathrm{Pt} / \mathrm{Co} / \mathrm{Pt}$ film showing perpendicular magnetization ( $\mathrm{z}$ axis), a DC is injected along the $\mathrm{x}$ direction while the anomalous Hall voltage is measured along the y direction. The fs laser beam perpendicular to the film plane is swept along the $\mathrm{x}$ axis to perform the sweeping beam measurement, while for the static beam measurement; the laser spot is shined on the $\mathrm{x}$ axis at a fixed position about $40 \mu \mathrm{m}$ from the center of the Hall cross.

$R_{A H E}$ is the anomalous Hall coefficient ${ }^{24,25}$ and $t$ is the thickness of the Hall cross. The studied Hall cross has a resistance of $R=1197 \Omega$. Its typical value of the anomalous Hall voltage is $V_{\mathrm{AHE}}=0.18 \mathrm{mV}$ for an applied current of $I=0.75 \mathrm{~mA}$.

The current density used in our experiment is $30 \mathrm{MA} / \mathrm{m}^{2}$. This value is small compared to the one used for magnetization switching by current-induced spin-orbit torque, which is of the order of $0.3 \mathrm{TA} / \mathrm{m}^{2}$. Thus, a direct current-induced switching is unlikely to happen. ${ }^{26,27}$

For the optical excitation, we use a Ti: sapphire fs-laser with a $5-\mathrm{kHz}$ repetition rate, a wavelength of $800 \mathrm{~nm}$, and pulse duration of $35 \mathrm{fs}$. The Gaussian beam spot is focused with a FWHM of approximately $60 \mu \mathrm{m}$. A quarter-wave plate is used to create the circular polarization of the beam, thereby providing a spin angular momentum of $+\hbar$ and $-\hbar$ for a right- $(\sigma+)$ and left- $(\sigma-)$ handed circularly polarized photon, respectively.

To optically switch the magnetization in the Hall cross, two approaches are used. For both approaches, before illumination, the sample is saturated under an external magnetic field applied along the $\mathrm{z}$ axis to quantify the Hall voltage for the two saturated magnetic states $\vec{M} \hat{e}_{\mathrm{z}}$ or $-\vec{M} \hat{e}_{\mathrm{z}}$. In the first approach called sweeping beam measurement, the laser beam is swept over the length of the magnetic wire along the $\mathrm{x}$ axis across the Hall cross. In the second approach called static beam measurement, the pulsed laser is maintained at a fixed position where the center of the beam is about $40 \mu \mathrm{m}$ from the center of the Hall cross. In both cases during optical excitations, no magnetic field is applied, and the anomalous Hall voltage is measured continuously, thus indicating the magnetic state in the central area of the Hall cross. A decrease in the anomalous Hall voltage could also be due to a change in the Hall resistivity, induced by the temperature increase. However, by adding an external magnetic field to the laser heated cross, the anomalous Hall voltage does not change compared to the one of the unheated cross. This excludes a significant change of the Hall resistivity.
As mentioned in the introduction, it is important to demonstrate not only the all-optical switching effect, but also its helicity dependence. Indeed, the switching of the central and active area of the Hall cross could also arise from domain formation by dipolar interaction, as this leads to a reduction of stray fields. Thus, a pure optical heating in the center of the cross could result in a switching of magnetization in this region of the cross, whereas the outer parts would remain unswitched. This heat induced domain creation would be irreversible and mainly helicity independent; thus, it can be excluded by proving the pronounced helicity dependence of our measurement. Hence, shown in Fig. 2 are sweeping measurements in all four possible configurations of light helicity and initial magnetization orientation. The sweeping speed of the laser beam is $40 \mu \mathrm{m} / \mathrm{s}$, and its power is 0.95 $\mathrm{mW}$.

The laser beam is swept from positions $-100 \mu \mathrm{m}$ to $100 \mu \mathrm{m}$. The zero position corresponds to the center of the Hall cross. As can be seen in Fig. 2(a), sweeping with leftcircularly polarized beam over the Hall cross switches the magnetization to the up state $\left(\overline{V_{\text {Hall }}}=1\right)$ independently of the initial state. Conversely for right-circularly polarized beam (Fig. 2(b)) the final state is down $\left(\overline{V_{\text {Hall }}}=-1\right)$. This undoubtedly demonstrates the helicity dependence of the

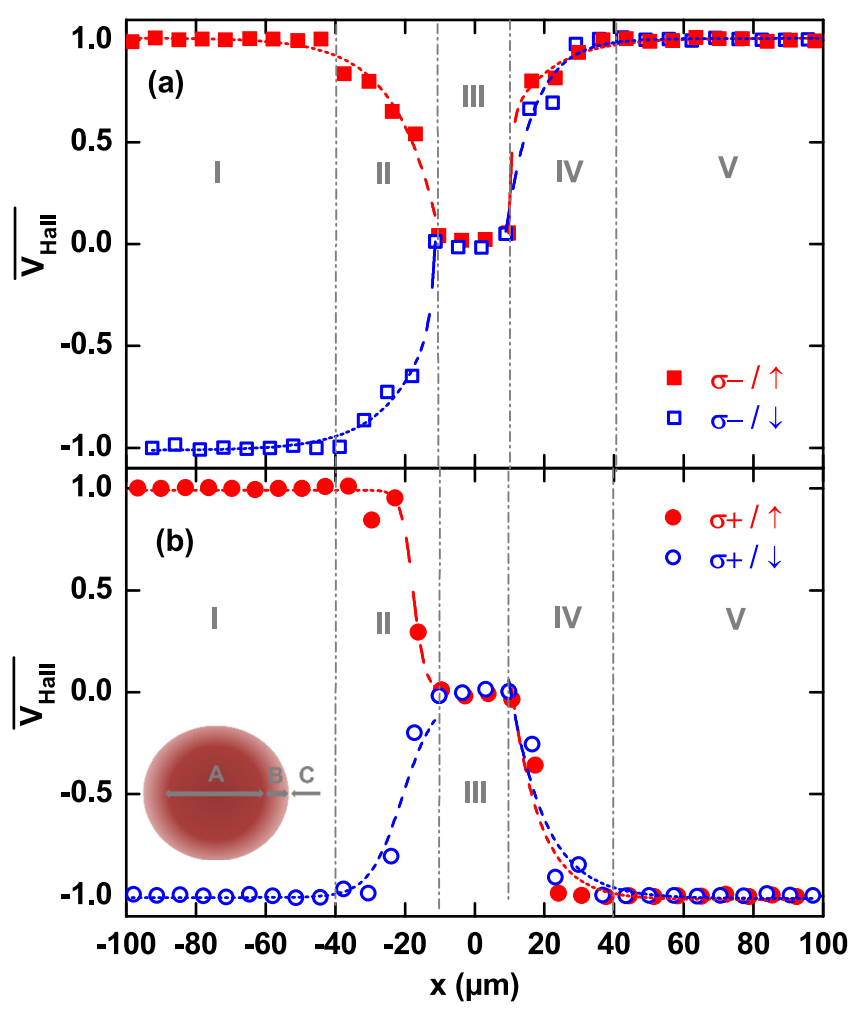

FIG. 2. Normalized Hall voltage $\overline{V_{\text {Hall }}}$ as a function of the position of the beam $\mathrm{x}$ swept where (a) corresponds to left-handed circular polarization $(\sigma-)$ and $(b)$ to right-handed circular polarization $(\sigma+)$. The values 1 and -1 of the $\overline{V_{\text {Hall }}}$ are the saturation of magnetization values. The laser beam is swept over the whole $\mathrm{x}$ axis of Hall cross from position $-100 \mu \mathrm{m}$ to $100 \mu \mathrm{m}$, with a sweeping speed of $40 \mu \mathrm{m} / \mathrm{s}$ and a power of $0.95 \mathrm{~mW}$. The experiment is repeated for both positive and negative saturation of the magnetization. The dashed lines are guides to the eyes. Shown in the inset is the schematic presentation of the different areas of the laser spot: "A" where multiple domains are obtained (approximately $80 \mu \mathrm{m}$ diameter); "B" where AO-HDS is obtained ( $2-3 \mu \mathrm{m}$ size); " $\mathrm{C}$ " where no change of magnetization is induced. 
observed all-optical switching process. The shapes of the curves in Fig. 2 can be understood considering that the beam can be decomposed into regions of different intensity (as described in previous studies ${ }^{4,11}$ ). In the central part of the laser spot, the intensity is sufficiently high to completely demagnetize the layer, whereas AO-HDS takes place for a narrow ring of the optimum intensity called AOS ring. When the beam is centered in region I (see Fig. 2), the laser beam is far from the Hall cross and has no effect on its magnetization. In region II, the absolute value of the Hall voltage is dropping as the first part of the AOS ring and the inner circle of the laser beam are entering the center of Hall cross. No switching is measured in the transition between regions I and II. The drop of Hall voltage in region II is probably due to thermal demagnetization or to a gradual presence of multiple domains in the cross. In contrast, in region III, the beam center is well aligned on the Hall cross and the presence of multiple domains in it leads to a zero plateau of the Hall voltage. In region IV, the signature of the helicity of the beam starts to appear as the second part of the AOS ring moves over the Hall cross area. Independently from the initial saturation, the magnetization switches gradually to up for $\sigma-$ and down for $\sigma+$ light, until reaching a complete switching when the beam center is in region $\mathrm{V}$.

Hence, this electrical measurement via the anomalous Hall effect is a clear demonstration of AO-HDS in $\mathrm{Pt} / \mathrm{Co} / \mathrm{Pt}$ based Hall cross, but it is observed after the sample is demagnetized by the center of the beam. This also enables the estimation of the diameter of the inner circle of the beam spot to be about $80 \mu \mathrm{m}$ for a beam power of $0.95 \mathrm{~mW}$.

For the power and the sweeping speed described in Fig. 2, a full all-optical switching is obtained. However, decreasing the power absorbed by the Hall cross leads to an incomplete switching with an additional stochastic contribution. This decrease in the number of absorbed photons can be realized by either decreasing the laser power and/or increasing the sweeping speed (Fig. 3). Note that those two ways are very different, since in one case the maximum intensity of each pulse is tuned, but the number and density of pulses stay constant, while in the second case, the number of pulses per unit area changes. When the sweeping speed increases from $40 \mu \mathrm{m} / \mathrm{s}$ to $2500 \mu \mathrm{m} / \mathrm{s}$, the distance between the two consecutive pulses increases from $8 \mathrm{~nm}$ to $0.5 \mu \mathrm{m}$, which remains low compared to the spot size that is about $80 \mu \mathrm{m}$. For both types of measurements, in order to take into account the stochastic contribution, all sweeping beam measurements in Fig. 3 were performed 20 times with a reset of the magnetization to its initial state after each sweep. For each individual measurement, the switching ratio $\tau$, which we define as the relative change of the anomalous Hall voltage in comparison to the full switching, is determined (a value of $\tau=100 \%$ corresponds to complete and deterministic switching). The values of the switching ratio given in Fig. 3 are arithmetic means of these results, and the value of the standard deviation is given by the length of the error bars.

In Fig. 3(a), the switching ratio is presented as a function of the laser power for various sweeping speeds. Only one combination of initial saturation and circular polarization is shown. We clearly observe a threshold power below which the laser does not affect the magnetization, and above which AO-HDS is observed. This threshold power is
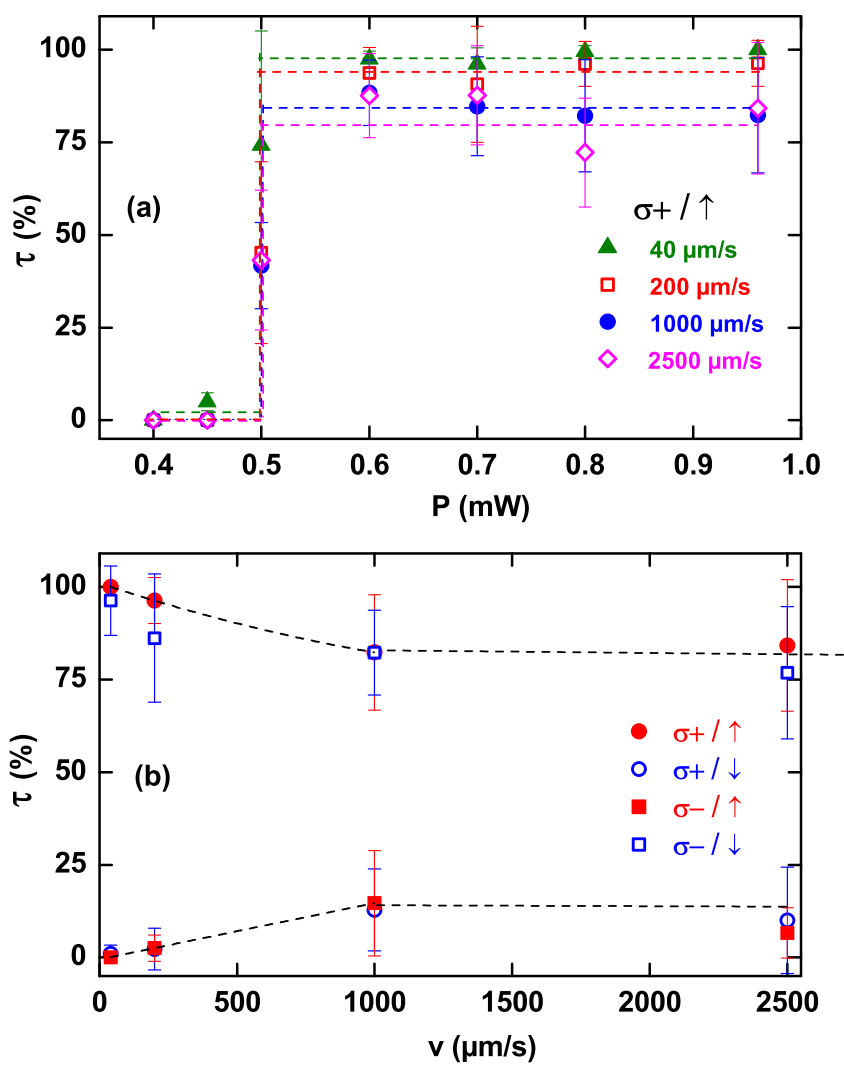

FIG. 3. (a) Switching ratio $\tau$ as a function of the laser power $P$ for different sweeping speeds. The initial saturation up with $\sigma+$ sweeping is exemplarily showed. (b) Switching ratio $\tau$ as a function of the swept beam's speed $v$ with a power of $0.95 \mathrm{~mW}$ for the four different combinations of circular polarization and initial saturation. The dashed lines are guides to the eyes.

independent of the sweeping speed at least in the range from $40 \mu \mathrm{m} / \mathrm{s}$ to $2500 \mu \mathrm{m} / \mathrm{s}$. Above the threshold power, a $100 \%$ switching is observed only for the lower sweeping speed $(40 \mu \mathrm{m} / \mathrm{s})$, whereas as the sweeping speed increases the switching ratio decreases and seems to saturate around $80 \%$. As the ratio decreases, the standard deviation increases, meaning that the process is increasingly stochastic. Note that this switching ratio seems to be dependent only weakly on the laser power above the threshold power.

Figure 3(b) shows the evolution of the switching ratio as a function of the sweeping speed for the four different combinations of circular polarization and initial saturation, with a laser power of $0.95 \mathrm{~mW}$ (largely above the threshold power). First, we can see that the expected symmetric behavior is observed. The helicity dependence of the all-optical switching process is conserved also for the highest measured sweeping speed of $2500 \mu \mathrm{m} / \mathrm{s}$. In fact, for a given saturation, the difference in the switching ratio between the two circular polarizations is larger than $75 \%$ for all sweeping speeds. For sweeping speeds higher than $200 \mu \mathrm{m} / \mathrm{s}$, the switching ratio drops by 10\%-20\% compared to the slowest speed of $40 \mu \mathrm{m} /$ $\mathrm{s}$, and at the same time, the standard deviation increases. This behavior could be explained by the persistent formation of multiple magnetic domains within the Hall cross area. The formation of these domains might be due to the fact that less pulses interact with the Hall cross for high sweeping speeds, indicating that the AO-HDS of the entire Hall cross is a cumulative process. 


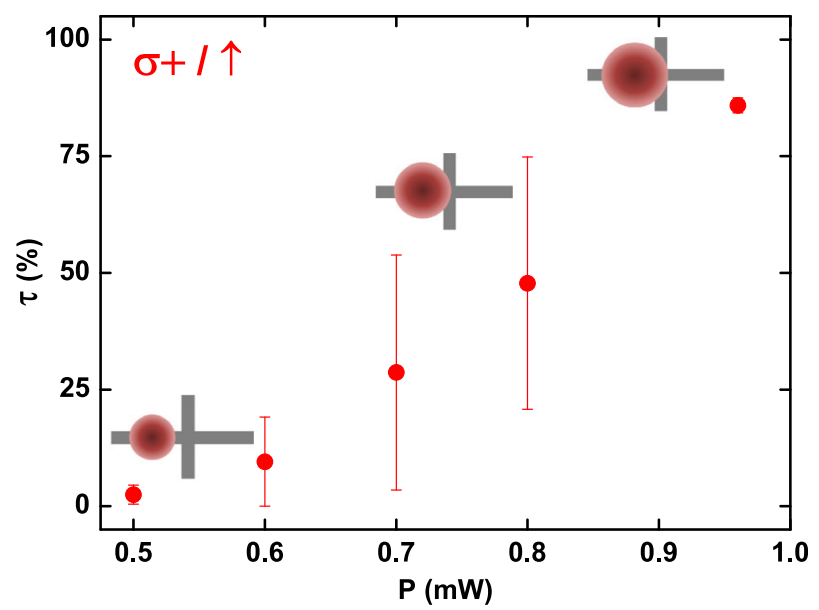

FIG. 4. Switching ratio $\tau$ as a function of the laser power $P$ for the static beam measurement. The center of the beam spot is kept fixed at a position approximately $40 \mu \mathrm{m}$ from the Hall cross center. Shown in the inset is the schematic evolution of the diameter of the all-optical switching ring.

To compare with sweeping beam measurements, we investigated static beam measurements as they provide insight into the switching process and a better integration of the all-optical switching into spintronic devices. Figure 4 shows such a static beam measurement where the switching ratio is measured as a function of the laser power for a fixed laser beam center position of approximately $40 \mu \mathrm{m}$ from the Hall cross center. The switching ratio is measured as the beam is on, to avoid demagnetization effects due to cooling when the beam is turned off. The rest of the measurement procedure (averaging over 10 measurements) is similar to that used for the sweeping beam measurement in Fig. 3. Again, we show the combination of initial saturation up and $\sigma+$ polarization. In this geometry, when the laser power increases, both the maximum laser power in the center of the laser spot and the size of the spot increase.

As can be seen from Fig. 4, no switching occurs for the minimum power of $0.5 \mathrm{~mW}$ as the laser power at the position of the Hall cross is too low to influence the magnetization. For powers from 0.6 to $0.8 \mathrm{~mW}$, the switching ratio increases gradually as the AOS ring gets centered on the Hall cross. In addition to partial switching, a significant stochastic component of the switching process appears, which is manifested in the large standard deviation. This might indicate that heatinduced magnetic domain fluctuations might play an important role for these laser power values. For a power of 0.95 $\mathrm{mW}$, a reliable switching of about $90 \%$ is achieved with a small standard deviation. The fact that only $90 \%$ switching ratio is reached in the static beam measurement could be due to the small width of the AOS ring, which is not covering the entire Hall cross area. This behavior is similar to the one of the sweeping beam measurement in Fig. 3(b), which is a hint that a certain number of photons are needed to obtain a reliable all-optical switching. Thus, a small area of the Hall cross remains un-switched. The helicity dependence has been verified by testing the other combinations of initial saturation and circular polarization.

In conclusion, we demonstrated all-optical helicity-dependent switching in a ferromagnetic $\mathrm{Pt} / \mathrm{Co} / \mathrm{Pt}$ film patterned into Hall cross to allow electrical measurement of the magnetization by the anomalous Hall voltage. Two methods were used: either the femtosecond laser beam was swept over the Hall cross or kept static close to the center of the cross. Both approaches indicate that a threshold power needs to be overcome to observe AO-HDS. By increasing the sweeping speed, the switching becomes incomplete, thus indicating that the AO-HDS of the entire Hall cross is a cumulative process. Those results tend to indicate that dynamics at different time scales are taking place. From a technological point of view, our studies show a first integration of AO-HDS of ferromagnetic layers into spintronic devices, by storing the circular polarization of the light in the remanence state of the magnetization. The helicity dependence plays a major role in this integration, as it enables a full and reproducible all-optical writing and erasing of data without applying any external magnetic field.

We would like to thank G. Lengaigne for technical assistance with lithography process. This work was supported by the ANR-NSF Project, ANR-13-IS04-0008-01, "COMAG" by the ANR-Labcom Project LSTNM, by the European Project (OP2M FP7-IOF-2011-298060) and by the Université de la Grande Region (UniGR funded P. Pirro Post-Doc). Experiments were performed using equipment from the TUBE - Daum funded by FEDER (EU), ANR, the Region Lorraine, and Grand Nancy.

${ }^{1}$ C. D. Stanciu, F. Hansteen, A. V. Kimel, A. Kirilyuk, A. Tsukamoto, A. Itoh, and T. Rasing, Phys. Rev. Lett. 99, 047601 (2007).

${ }^{2}$ A. Kirilyuk, A. V. Kimel, and T. Rasing, Rev. Mod. Phys. 82, 2731-2784 (2010).

${ }^{3}$ I. Radu, K. Vahaplar, C. Stamm, T. Kachel, N. Pontius, H. A. Durr, T. A. Ostler, J. Barker, R. F. Evans, R. W. Chantrell et al., Nature 472, 205-208 (2011).

${ }^{4}$ T. A. Ostler, J. Barker, R. F. L. Evans, R. W. Chantrell, U. Atxitia, O. Chubykalo-Fesenko, S. El Moussaoui, L. Le Guyader, E. Mengotti, L. J. Heyderman et al., Nat. Commun. 3, 666 (2012).

${ }^{5}$ K. Vahaplar, A. M. Kalashnikova, A. V. Kimel, S. Gerlach, D. Hinzke, U. Nowak, R. Chantrell, A. Tsukamoto, A. Itoh, A. Kirilyuk et al., Phys. Rev. B 85, 104402 (2012).

${ }^{6}$ A. R. Khorsand, M. Savoini, A. Kirilyuk, A. V. Kimel, A. Tsukamoto, A. Itoh, and T. Rasing, Phys. Rev. Lett. 108, 127205 (2012).

${ }^{7}$ C. E. Graves, A. H. Reid, T. Wang, B. Wu, S. de Jong, K. Vahaplar, I. Radu, D. P. Bernstein, M. Messerschmidt, L. Muller et al., Nat. Mater. 12, 293-298 (2013).

${ }^{8}$ S. Mangin, M. Gottwald, C.-H. Lambert, D. Steil, V. Uhlir, L. Pang, M. Hehn, S. Alebrand, M. Cinchetti, G. Malinowski et al., Nat. Mater. 13, 286-292 (2014).

${ }^{9}$ S. Alebrand, M. Gottwald, M. Hehn, D. Steil, M. Cinchetti, D. Lacour, E. E. Fullerton, M. Aeschlimann, and S. Mangin, Appl. Phys. Lett. 101, 162408 (2012).

${ }^{10}$ C. Schubert, A. Hassdenteufel, P. Matthes, J. Schmidt, M. Helm, R. Bratschitsch, and M. Albrecht, Appl. Phys. Lett. 104, 082406 (2014).

${ }^{11}$ C. H. Lambert, S. Mangin, B. S. D. C. S. Varaprasad, Y. K. Takahashi, M. Hehn, M. Cinchetti, G. Malinowski, K. Hono, Y. Fainman, M. Aeschlimann et al., Science 345, 1337-1340 (2014).

${ }^{12}$ C. Caspers, D. Yoon, M. Soundararajan, and J.-P. Ansermet, New J. Phys. 17, 022004 (2015).

${ }^{13}$ E. Beaurepaire, J. Merle, A. Daunois, and J. Bigot, Phys. Rev. Lett. 76, 4250-4253 (1996).

${ }^{14}$ J.-Y. Bigot, M. Vomir, and E. Beaurepaire, Nat. Phys. 5, 515-520 (2009).

${ }^{15}$ G. Malinowski, F. Dalla Longa, J. H. H. Rietjens, P. V. Paluskar, R. Huijink, H. J. M. Swagten, and B. Koopmans, Nat. Phys. 4, 855-858 (2008). ${ }^{16}$ C. Chappert, A. Fert, and F. Nguyen Van Dau, Nat. Mater. 6, 813-823 (2007).

${ }^{17}$ S. A. Wolf, D. D. Awschalom, R. A. Buhrman, J. M. Daughton, S. von Molnar, M. L. Roukes, A. Y. Chtchelkanova, and D. M. Treger, Science 294, 1488-1495 (2001).

${ }^{18}$ A. D. Kent and D. C. Worledge, Nat. Nanotechnol. 10, 187-191 (2015). 
${ }^{19}$ S. Parkin and S.-H. Yang, Nat. Nanotechnol. 10, 195-198 (2015).

${ }^{20}$ M. H. Kryder, E. C. Gage, T. W. McDaniel, W. A. Challener, R. E. Rottmayer, G. P. Ju, Y. T. Hsia, and M. F. Erden, Proc. IEEE 96, 1810-1835 (2008).

${ }^{21}$ B. C. Stipe, T. C. Strand, C. C. Poon, H. Balamane, T. D. Boone, J. A. Katine, J. L. Li, V. Rawat, H. Nemoto, A. Hirotsune et al., Nat. Photonics 4, 484-488 (2010).

${ }^{22}$ S. Mangin, D. Ravelosona, J. A. Katine, M. J. Carey, B. D. Terri, and E. E. Fullerton, Nat. Mater. 5, 210 (2006).
${ }^{23}$ L. He, J.-Y. Chen, J.-P. Wang, and M. Li, Appl. Phys. Lett. 107, 102402 (2015).

${ }^{24}$ J. M. Luttinger, Phys. Rev. 112, 739-751 (1958).

${ }^{25}$ L. Berger, Phys. Rev. B 2, 4559-4566 (1970).

${ }^{26}$ L. Liu, O. J. Lee, T. J. Gudmundsen, D. C. Ralph, and R. A. Buhrman, Phys. Rev. Lett. 109, 096602 (2012).

${ }^{27}$ J.-C. Rojas-Sanchez, P. Laczkowski, J. Sampaio, S. Collin, K. Bouzehouane, N. Reyren, H. Jaffres, A. Mougin, and J.-M. George, Appl. Phys. Lett. 108, 082406 (2016). 